Berufsverband der Deutschen Urologen e.V.

Urologe 2014 · 53:141

DOI 10.1007/s00120-013-3393-0

๑) Springer-Verlag Berlin Heidelberg 2014
Redaktion

W. Bühmann,Wenningstedt/Sylt

\title{
++4 URO-Telegramm
}

\section{Entscheidende Reformen sind nötig}

"Dringliche Überweisung" für schnelle Termine beim Facharzt lösen die Probleme bei der Versorgung nicht

Die Initiative der dritten schwarzroten Koalition, Wartezeiten bei Facharztterminen zu reduzieren, ist purer Populismus, weil es sich vorrangig um ein subjektiv empfundenes Problem handelt. In der Realität sieht dies ganz anders aus. In der Zusammenarbeit zwischen Hausarzt und Facharzt, insbesondere in der Akutversorgung gibt es so gut wie keine Wartezeiten. Deshalb ist es auch in Zukunft wichtig und im Interesse der Patienten, dass die Dringlichkeit einer Behandlung primär der Arzt feststellt und nicht der Patient, schon gar nicht Technokraten in einer Servicestelle. Wir brauchen nicht mehr Bürokratie und Administration, sondern endlich auch den Freiraum und die Rechtssicherheit sektorübergreifend (Verzahnung ambulant und stationär) arbeiten zu können. Die Kliniken für die fachärztliche Versorgung zu öffnen, wenn es kurzfristig keinen Termin beim niedergelassenen Arzt gibt, ist unsinnig. Kliniken sind schon heute überlastet und wir haben in Deutschland im Vergleich zum europäischen Ausland sensationell kurze Wartezeiten. Dies ist der Situation geschuldet, dass wir in der Bundesrepublik noch eine wohnortnahe, flächendeckende Facharztversorgung durch niedergelassene Ärzte haben. Ambulante und stationäre fachärztliche Versorgung ergänzen sich und sind bislang der Garant für eines der besten Gesundheitssysteme der Welt.
Vielmehr sind entscheidende Reformen notwendig, denn wir stoßen auch in der fachärztlichen Versorgung auf einen Ärztemangel. Dieser wird in den nächsten Jahren aufgrund der Alterung der Gesellschaft noch eklatanter. Da hilft auch eine Überweisung 1. und 2. Klasse, wie vom Präsidenten der Bundesärztekammer vorgeschlagen, wenig. Wir brauchen neue Strukturen und bessere Rahmenbedingungen. In einer Welt der Budgetierung und einer Deckelung der Leistungen wird man dem Mehrbedarf nicht gerecht. Der große Bedarf und damit die Herausforderung in der ambulanten vertragsärztlichen Versorgung ist der Kassenpatient und nicht der Privatpatient. Der Anteil der Privatpatienten macht durchschnittlich lediglich $10 \%$ in einer Kassenarztpraxis aus. Diese führen nicht zur Verdrängung der Termine bzw. lösen keine lange Wartezeiten für Kassenpatienten aus.

Demografie und Ärztemangel sind u.a. die Herausforderung unserer Gesellschaft in puncto Gesundheitspolitik. Vermehrte Bürokratie und Administration sind da die falschen Antworten der Politik und lenken nur von den System- und Strukturschwächen ab. Allerdings würde auch mehr Eigenverantwortung des Bürgers in unserer Gesellschaft nichts schaden, gerade in Sachen Gesundheit. Unser Gesundheitssystem und damit auch eine Facharztpraxis ist kein Selbstbedienungsladen.

Dr. med. Axel Schroeder

Präsident des Berufsverbandes der Deutschen Urologen e.V. Vorstand des Spitzenverbandes Fachärzte Deutschland Neumünster

\section{Unruhe mit IGeL garantiert!}

Selbstzahlerleistungen werden auch 2014 ein beliebtes Thema der Medien und Kassen sein. Bei vielen Ärzten gibt es noch Defizite im Umgang mit IGeL. Jetzt ist es an der Zeit, diese offensiv auszugleichen.

Individuelle Gesundheitsleistungen (IGeL) und der verantwortungsvolle Umgang mit innen im Praxisalltag wurden im zu Ende gehenden Jahr wieder heftig diskutiert.

Ein Anlass war mit Sicherheit das im Februar in Kraft getretene Patientenrechtegesetz, das niedergelassenen Vertragsärzten oktroyiert, an Selbstzahlerleistungen interessierten Patienten bereits im Angebotsgespräch - und damit vor Beginn der Behandlung - die voraussichtlichen Kosten zu beziffern.

Das impliziert aber bereits, dass die Ärzte im Praxisalltag neben der gesetzlich geforderten Aufklärungspflicht auch der Pflicht nachkämen, vor dem Wunsch-Eingriff einen schriftlichen Behandlungsvertrag vorzulegen, der - basierend auf der GOÄ - Art und Umfang der Leistung ersichtlich und mit der Unterschrift des Patienten die Inanspruchnahme auch verpflichtend macht.

\section{Defizite gibt es bei der Patientenaufklärung}

Wie nicht anders zu erwarten, hakt es im oft hektischen Hausarztalltag - dieser darf aber nicht als Entschuldigungsgrund herangezogen werden - bereits an der Aufklärung der Patienten.

Wie das Wissenschaftliche Institut der AOK (WIdO) in einer Umfrage zu Jahresbeginn herausgefunden hatte, hatten nur 46,2 Prozent der teilnehmenden Patienten eine Aufklärung erfahren bezüglich der Zuverlässigkeit der im Rahmen ihres nachgefragten Selbstzahlerangebotes angewandten Untersuchungsmethode. 39 Prozent der Patienten gaben an, dies sei sogar gar nicht thematisiert worden.

Bleibt die Frage, wie niedergelassene Vertragsärzte ihre Praxisabläufe organisatorisch so optimieren können, dass sie alle gesetzlichen IGeL-Mindestanforderungen erfüllen.

Matthias Wallenfels

Erfolgreich in die
Nelbstständigkeit in der Urologie
der Zukunft
unkalkulierbares Risiko oder ungeahnte Chance?
Workshop am 21.03. - 22.03.2014 in Berlin
Informationen unter www.gesru.de

